\title{
INTERNAL CONSISTENCY AND THE INNER MODEL HYPOTHESIS
}

\author{
SY-DAVID FRIEDMAN
}

There are two standard ways to establish consistency in set theory. One is to prove consistency using inner models, in the way that Gödel proved the consistency of GCH using the inner model $L$. The other is to prove consistency using outer models, in the way that Cohen proved the consistency of the negation of $\mathrm{CH}$ by enlarging $L$ to a forcing extension $L[G]$.

But we can demand more from the outer model method, and we illustrate this by examining Easton's strengthening of Cohen's result:

Theorem 1. (Easton's Theorem) There is a forcing extension $L[G]$ of $L$ in which GCH fails at every regular cardinal.

Assume that the universe $V$ of all sets is rich in the sense that it contains inner models with large cardinals. Then what is the relationship between Easton's model $L[G]$ and $V$ ? In particular, are these models compatible, in the sense that they are inner models of a common third model? If not, then the failure of GCH at every regular cardinal is consistent only in a weak sense, as it can only hold in universes which are incompatible with the universe of all sets. Ideally, we would like $L[G]$ to not only be compatible with $V$, but to be an inner model of $V$.

We say that a statement is internally consistent iff it holds in some inner model, under the assumption that there are inner models with large cardinals. By specifying what large cardinals are required, we obtain a new type of consistency result. Let $\mathrm{Con}(\mathrm{ZFC}+\varphi)$ stand for "ZFC $+\varphi$ is consistent" and Icon $(\mathrm{ZFC}+\varphi)$ stand for "there is an inner model of $\mathrm{ZFC}+\varphi$ ". A typical consistency result takes the form

$$
\mathrm{Con}(\mathrm{ZFC}+\mathrm{LC}) \rightarrow \mathrm{Con}(\mathrm{ZFC}+\varphi)
$$

where LC denotes some large cardinal axiom. An internal consistency result takes the form

$$
\mathrm{Icon}(\mathrm{ZFC}+\mathrm{LC}) \rightarrow \operatorname{Icon}(\mathrm{ZFC}+\varphi) .
$$


Thus a statement $\varphi$ is internally consistent relative to large cardinals iff $\operatorname{Icon}(\mathrm{ZFC}+\varphi)$ follows from Icon $(\mathrm{ZFC}+\mathrm{LC})$ for some large cardinal axiom LC.

A statement can be consistent without being internally consistent relative to large cardinals. An example is the statement that there are no transitive models of ZFC, which fails in any inner model, assuming there are inner models with inaccessible cardinals. Another example is:

\section{For each infinite regular cardinal $\kappa$ there is a nonconstructible subset of $\kappa$ whose proper initial segments are constructible.}

This can be forced over $L$, but does not hold in any inner model, assuming the existence of $0^{\#}$.

If the consistency of a statement without parameters is shown using set forcing, then it is usually easy to prove its internal consistency relative to large cardinals; some examples are mentioned below. But this is not the case for statements that contain uncountable parameters or for statements whose consistency is shown through the use of class forcing. In these latter cases, questions of internal consistency and of internal consistency strength can be quite interesting, as we shall now see.

\section{Easton's theorem revisited}

Let Reg denote the class of infinite regular cardinals and Card the class of all infinite cardinals. An Easton function is a class function $F: \operatorname{Reg} \rightarrow$ Card such that:

For all $\kappa \leq \lambda$ in Reg: $F(\kappa) \leq F(\lambda)$.

For all $\kappa \in \operatorname{Reg}$ : $\operatorname{cof}(F(\kappa))>\kappa$.

Easton showed that if $F$ is an Easton function in $L$, then there is a cofinalitypreserving class forcing extension $L[G]$ of $L$ in which $2^{\kappa}=F(\kappa)$ for all regular $\kappa$. We say that the model $L[G]$ realises the Easton function $F$.

Which Easton functions in $L$ can be realised in an inner model? The following are some partial results, obtained jointly with Pavel Ondrejović $([8])$.

Theorem 2. Suppose that $0^{\#}$ exists and $F$ is an Easton function in $L$ which is L-definable using parameters which are countable in $V$. Then there exists an inner model with the same cofinalities as $L$ in which $2^{\kappa}=F(\kappa)$ for each infinite regular $\kappa$. 
Corollary 3. The statement

$$
2^{\kappa}=\kappa^{++} \text {for all infinite regular } \kappa
$$

is internally consistent relative to the existence of $0^{\#}$.

Internal consistency can sometimes be obtained when we allow uncountable parameters.

Theorem 4. Assume that $0^{\#}$ exists and $\kappa$ is a regular uncountable cardinal. Then there is an inner model with the same cofinalities as $L$ in which $G C H$ holds below $\kappa$ but fails at $\kappa$.

How badly can GCH fail in an inner model? The proof of the following uses the existence of a gap-1 morass in $L\left[0^{\#}\right]$.

Theorem 5. Assume that $0^{\#}$ exists and $\kappa$ is a regular uncountable cardinal. Then there is an inner model with the same cofinalities as $L$ in which $2^{\kappa}=$ $\left(\kappa^{+}\right)^{V}$.

GCH fails below $\kappa$ in the inner model of Theorem 5 . If we require that GCH hold below $\kappa$ we obtain a weaker conclusion:

Theorem 6. Assume that $0^{\#}$ exists, $\kappa$ is a regular uncountable cardinal and $\alpha$ is less than $\left(\kappa^{+}\right)^{V}$. Then there is an inner model with the same cofinalities as $L$ in which $G C H$ holds below $\kappa$ and $2^{\kappa}>\alpha$.

Conjecture. Assume the existence of $0^{\#}$. Then an $L$-definable Easton function $F$ can be realised in an inner model $M$ (having the same cofinalities as $L)$ iff it satisfies:

$$
F(\kappa)<\left(\kappa^{++}\right)^{V} \text { for all } \kappa \in \operatorname{Reg}^{L} .
$$

The singular cardinal hypothesis

The analog of Cohen's result for the singular cardinal hypothesis is:

Theorem 7. (Gitik [11]) Suppose that $K$ is an inner model satisfying $G C H$ which contains a totally measurable cardinal $\kappa$, i.e., a cardinal $\kappa$ of Mitchell order $\kappa^{++}$. Then there is a generic extension $K[G]$ of $K$ in which $\kappa$ is a singular strong limit cardinal and GCH fails at $\kappa$.

Gitik also shows that a totally measurable cardinal is necessary. Now consider the following weak analogue of Easton's result for the singular cardinal hypothesis:

(Global Gitik) GCH fails on a proper class of singular strong limit cardinals.

The proof of the previous theorem shows: 
Theorem 8. Suppose that $K$ is an inner model satisfying GCH which contains a proper class of totally measurable cardinals. Then there is a generic extension $K[G]$ of $K$ in which Global Gitik holds.

Is Global Gitik internally consistent relative to large cardinals? In analogy to Easton's theorem, we might expect to show that the generic extension $K[G]$ of Theorem 8 can be obtained as an inner model. This is however not true for the natural choice of $K$. The following work is joint with Tomáš Futáš $([7])$.

Theorem 9. Suppose that there is a \# for a proper class of totally measurable cardinals and let $K$ be the "natural" inner model with a class of totally measurable cardinals. ( $K$ is obtained by taking the least iterable mouse $m$ with a measurable limit of totally measurable cardinals and iterating its top measure out of the universe.) Then there is no inner model of the form $K[G]$, where $G$ is generic over $K$, in which Global Gitik holds.

On the other hand, it is possible to choose $K$ differently, so as to witness the internal consistency relative to large cardinals of Global Gitik:

Theorem 10. Suppose that there is an inner model containing a measurable limit $\kappa$ of totally measurable cardinals, where $\kappa$ is countable in $V$. Then there is an inner model in which Global Gitik holds.

What is the internal consistency strength of Global Gitik, i.e., what large cardinal hypothesis must hold in some inner model to obtain an inner model of Global Gitik?

Theorem 10 provides an upper bound. In analogy to the proof of the internal consistency relative to $0^{\#}$ of Easton's result, one would expect that a \# for a proper class of totally measurables, a weaker assumption, would also suffice.

But unlike with Easton's result, it is possible that the internal consistency strength of Global Gitik is the same as its external consistency strength, i.e., just a proper class of totally measurable cardinals, without its \#. The next result is an example of this unexpected phenomenon.

A cardinal $\kappa$ is Jonsson iff every structure of cardinality $\kappa$ for a countable language as a proper substructure of cardinality $\kappa$. By work of Mitchell [12], if there is a singular Jonsson cardinal then there is an inner model with a measurable cardinal. Conversely, if $M$ is an inner model with a measurable cardinal then $M[G]$ has a singular Jonsson cardinal, when $G$ is Prikry generic over $M$. But in fact an inner model with a singular Jonsson cardinal can be obtained inside $M$ : 
Theorem 11. Suppose that there is an inner model with a measurable cardinal. Then there is an inner model with a singular Jonsson cardinal.

This is proved as follows: Let $\kappa$ be measurable in an inner model $M$. Iterate $M$ using the measure on $\kappa$ to $M=M_{0} \supseteq M_{1} \supseteq \cdots$, and let $M^{*}$ be $M_{\omega}$. Then $\left\langle\kappa_{n} \mid n \in \omega\right\rangle$ produces a Prikry generic $G$ over $M^{*}$ and $M^{*}[G]$ is an inner model with a singular Jonsson cardinal.

Thus the internal consistency strength of a singular Jonsson cardinal is the same as its consistency strength, that of one measurable cardinal. Is the situation similar with Gitik's Theorem 7? I.e., can Con be replaced with Icon in the implication $\mathrm{Con}(\mathrm{ZFC}+$ there exists a totally measurable) $\rightarrow$ $\mathrm{Con}(\mathrm{ZFC}+\mathrm{GCH}$ fails at a singular strong limit)? Equivalently:

Question. Suppose that there is an inner model with a totally measurable cardinal. Then is there an inner model in which the GCH fails at a singular strong limit cardinal?

\section{Two more internal consistency results}

Katherine Thompson ([9]) and I have studied the global complexity of universal classes for certain types of structures. For a regular cardinal $\lambda$, we say that a poset $P$ omits $\lambda$ chains iff there is no order-preserving embedding of $\lambda$ into $P$. For a regular cardinal $\kappa \geq \lambda$, let $O(\kappa, \lambda)$ denote the collection of posets of cardinality $\kappa$ which omit $\lambda$ chains. Then the complexity of $O(\kappa, \lambda)$, written $K(\kappa, \lambda)$, is the smallest cardinality of a subset $S$ of $O(\kappa, \lambda)$ such that every element of $O(\kappa, \lambda)$ can be embedded into an element of $S$. Thompson and I obtained the following "high complexity" internal consistency result.

Theorem 12. Assume that $0^{\#}$ exists. Suppose that $F$ is an Easton function in $L$ which is $L$-definable without parameters. Also suppose that $\lambda$ is a parameter-free $L$-definable function which to each $L$-regular cardinal $\kappa>\omega$ associates a regular $L$-cardinal $\lambda(\kappa) \leq \kappa$. Then there is an inner model with the same cofinalities as $L$ in which $2^{\kappa}=F(\kappa)=K(\kappa, \lambda(\kappa))$ for each $L$-regular $\kappa>\omega$.

For $F$ and $\lambda$ as above, we also obtain the internal consistency of $2^{\kappa}=$ $F(\kappa)$ and $K(\kappa, \lambda(\kappa))=\kappa^{+}$for each $L$-regular $\kappa>\omega$ ("low complexity"). But we do not know if this statement is internally consistent relative to large cardinals if $\kappa^{+}$is replaced by $\kappa^{++}$.

The situation is similar concerning a joint result with Natasha Dobrinen ([2]). For cardinals $\kappa<\lambda, \kappa$ regular and uncountable, let $P_{\kappa}(\lambda)$ denote the set of subsets of $\lambda$ of cardinality less than $\kappa$. A result of Avraham-Shelah is 
that if $G$ is generic over $V$ for a ccc forcing that adds a real, then $P_{\kappa}(\lambda) \backslash V$ is stationary in $V[G]$ for all $\kappa<\lambda$. Dobrinen and I show:

Theorem 13. It is consistent relative to the existence of a proper class of $\omega_{1}$-Erdös cardinals that $G C H$ holds and $P_{\kappa}(\lambda) \backslash V$ is stationary in $V[G]$ for all regular $\kappa$ greater than $\omega_{1}$, where $G$ is generic over $V$ for $\omega_{1}$-Cohen forcing.

The property expressed in this theorem is internally consistent relative to a proper class of $\omega_{1}$-Erdős cardinals provided we restrict $\kappa$ to be a successor cardinal; otherwise the question is open and would appear to require at least the internal consistency of a proper class of Woodin cardinals.

\section{THE INNER MODEL HYPOTHESIS}

Recall that a statement is internally consistent iff it holds in some inner model. Therefore the meaning of internal consistency depends on what inner models exist. If we enlarge the universe, it is possible that more statements become internally consistent.

The inner model hypothesis asserts that the universe has been maximised with respect to internal consistency in the following sense: if a statement without parameters holds in an inner model of some outer model of $V$ (i.e., in a model compatible with $V$ ) then it already holds in an inner model of $V$. This is formalised by regarding $V$ as a countable transitive model of ZFC, taking the countable transitive models of ZFC of the same ordinal height which contain it as its outer models and taking the countable transitive models of ZFC of the same ordinal height contained in it as its inner models.

The strong inner model hypothesis, introduced later, has considerable large cardinal strength. This shows that a considerable part of our basic assumption, that of the internal consistency of large cardinals, is derivable from a natural absoluteness principle, the maximisation of internal consistency.

We next observe that the inner model hypothesis can be regarded as a second-order generalisation of:

Parameter-free Lévy-Shoenfield absoluteness. Suppose that $\varphi$ is a $\Sigma_{1}$ sentence true in an extension of $V$. Then $\varphi$ is true in $V$.

Recall that $\Sigma_{1}$ formulas are persistent in the sense that if such a formula is true in a transitive set, it is also true in all larger transitive sets. We 
consider persistent second-order formulas: A formula is persistently $\Sigma_{1}^{1}$ iff it is of the form

$\exists M(M$ is a transitive class and $M \vDash \psi)$,

where $\psi$ is first-order. We now regard $V$ as a model of (Gödel-Bernays) class theory, endowed with both sets and classes. By an outer model of $V$ we mean a model of class theory $V^{*}$, with the same ordinals as $V$, whose sets include the sets of $V$ and whose classes include the classes of $V$. Clearly if $V$ satisfies a persistent $\Sigma_{1}^{1}$ formula then so do all of its outer models.

Theorem 14. The following are equivalent:

(a) (Parameter-free persistent $\Sigma_{1}^{1}$ absoluteness). If a parameter-free $\Sigma_{1}^{1}$ formula is true in an outer model of $V$ then it is true in $V$.

(b) (Inner model hypothesis). If a first-order sentence is true in some model compatible with $V$ then it is true in some inner model of $V$.

Proof. (a) $\rightarrow$ (b): Suppose that the first-order sentence $\varphi$ is true in some model $M$ compatible with $V$. Then $M$ is an inner model of some outer model $V^{*}$ of $V$. The existence of $M$ can be expressed as a parameter-free $\Sigma_{1}^{1}$ formula, and therefore by (a), $\varphi$ holds in an inner model of $V$.

(b) $\rightarrow$ (a): Suppose that the parameter-free persistent $\Sigma_{1}^{1}$ formula $\varphi \equiv$ $\exists M(M$ is a transitive class and $M \vDash \psi)$ holds in some outer model $V^{*}$ of $V$. Then $\psi$ is true in some model compatible with $V$ and therefore by hypothesis, $\psi$ is true in some inner model of $V$. It follows that $\varphi$ holds in $V$.

Remark. The inner model hypothesis implies absoluteness for sentences which are $\Sigma_{2}$ over $H\left(\omega_{1}\right)$ (equivalently, for sentences which are $\Sigma_{3}^{1}$ in the sense of descriptive set theory). This is because by Lévy-Shoenfield absoluteness, such a sentence is true iff it is true in some inner model.

Theorem 15. (a) The inner model hypothesis implies that for some real $R$, $Z F C$ fails in $L_{\alpha}[R]$ for all ordinals $\alpha$. In particular, there are no inaccessible cardinals and the reals are not closed under \#.

(b) ([10]) The inner model hypothesis implies the existence of an inner model with measurable cardinals of arbitrarily large Mitchell order.

(c) ([10]) The consistency of the inner model hypothesis follows from the consistency of a Woodin cardinal.

Absolute parameters and the strong inner model hypothesis

How can we introduce parameters into the inner model hypothesis? The following result shows that inconsistencies arise without strong restrictions on the type of parameters allowed. 
Proposition 16. The inner model hypothesis with arbitrary ordinal parameters or with arbitrary real parameters is inconsistent.

Proof. With arbitrary ordinal parameters, inconsistency results from the fact that $\aleph_{1}$ can be countable in an outer model. To obtain an inconsistency with arbitrary real parameters, argue as follows. By (a) of Theorem 15, even the parameter-free version implies the existence of a real $R$ such that $\omega_{1}$ equals $\omega_{1}$ of $L[R]$. Then the statement " $\omega_{1}$ of $L[R]$ is countable" (with parameter $R$ ) holds in some outer model but not in any inner model.

So instead we consider absolute parameters, as in [6]. For a set $p$ and two transitive models $V_{0}, V_{1}$ containing $p$ as an element, we say that $p$ is absolute between $V_{0}$ and $V_{1}$ via the formula $\psi$ iff $\psi$ is a first-order formula without parameters which defines $p$ both in $V_{0}$ and in $V_{1}$.

Inner model hypothesis with absolute parameters Suppose that $p$ is absolute between $V$ and $V^{*}$, where $V^{*}$ is an outer model of $V$, and $\varphi$ is a first-order sentence with parameter $p$ which holds in an inner model of $V^{*}$. Then $\varphi$ holds in an inner model of $V$.

Theorem 17. ([10]) The inner model hypothesis with absolute parameters is inconsistent.

To obtain the strong inner model hypothesis, we restrict ourselves to absolute ordinal parameters.

Strong inner model hypothesis Suppose that the ordinal $\alpha$ is absolute between $V$ and $V^{*}$, where $V^{*}$ is an outer model of $V$, and $\varphi$ is a first-order sentence with parameter $\alpha$ which holds in an inner model of $V^{*}$. Then $\varphi$ holds in an inner model of $V$.

Remark. If above we assume that the sentence $\varphi$ holds not just in an inner model of $V^{*}$ but in $V^{*}$ itself, then in the conclusion we may demand that $\alpha$ be absolute between $V$ and an inner model of $V$ witnessing $\varphi$, via the same formula $\psi$ witnessing the absoluteness of $\alpha$ between $V$ and $V^{*}$. This is because we can replace the sentence $\varphi$ by: " $\varphi$ holds and $\alpha$ is defined by $\psi$ ".

Theorem 18. ([10]) The strong inner model hypothesis implies the existence of an inner model with a strong cardinal.

Remark. It is conjectured that core model theory can be extended from strong cardinals to Woodin cardinals, without any large cardinal assumptions. If this is the case, then the strong inner model hypothesis implies the existence of an inner model with a Woodin cardinal. 
Questions. 1. What is the exact consistency strength of the inner model hypothesis? 2. Is the strong inner model hypothesis consistent relative to large cardinals? If so, what is its consistency strength?

\section{REFERENCES}

[1] Beller, A., Jensen, R. and Welch, P. Coding the Universe, London Math Society Lecture Note Series, 47 (1982) Cambridge University Press.

[2] Dobrinen, N. and Friedman, S. Costationarity of the ground model, to appear.

[3] Friedman, S. New $\Sigma_{3}^{1}$ facts, Proceedings of the American Mathematical Society. Vol. 127, pp. 3707-3709, 1999.

[4] Friedman, S. Fine structure and class forcing, de Gruyter, 2000.

[5] Friedman, S. Generic $\Sigma_{3}^{1}$ absoluteness, Journal of Symbolic Logic, Vol. 69, No. 1, pp. 73-80, 2004.

[6] Friedman, S. Stable axioms of set theory, to appear, Proceedings of the Barcelona set theory year.

[7] Friedman, S. and Futáš, T. The internal consistency of the singular cardinal hypothesis, in preparation.

[8] Friedman, S. and Ondrejović, P. The internal consistency of Easton's theorem, in preparation.

[9] Friedman, S. and Thompson, K. High complexity for all regular uncountable cardinals, to appear.

[10] Friedman, S., Welch, P. and Woodin, W.H. On the consistency strength of the inner model hypothesis, in preparation.

[11] Gitik M. The negation of SCH from $o(\kappa)=\kappa^{++}$, Annals of Pure and Applied Logic 43, No.3, 1989.

[12] Mitchell, W. An introduction to inner models and large cardinals, a chapter for the Handbook of Set Theory.

[13] Zeman, M. Inner models and large cardinals, de Gruyter Series in Logic and Its Applications Vol.5, 2002. 